\title{
Electrode Mechanism Analysis by Linear Sweep Voltammetry. III. Reaction Orders, the Origin of the Response
}

\author{
VERNON D. PARKER
}

Laboratory for Organic Chemistry, Norwegian Institute of Technology, University of Trondheim, N-7034 Trondheim-NTH, Norway

The linear sweep voltammetry observables; $d E^{\mathrm{p}} / \mathrm{d}$ $\log v, \mathrm{~d} E^{\mathrm{p}} / \mathrm{d} \log C_{\mathrm{A}}$ and $\mathrm{d} E^{\mathrm{p}} / \mathrm{d} \log C_{\mathrm{X}}$, are intimately related to the reaction orders with respect to $B, A$ and $X$, respectively, where $A$ is the substrate, $B$ is the electrode-generated intermediate and $\mathrm{X}$ is a species reacting with $B$. In order to predict the response for any mechanism, it is only necessary to derive the rate equation (i). In (i), $C_{1}$ refers to the

Rate $=k_{\mathrm{obs}} C_{\mathrm{B}}^{\mathrm{b}} C_{\mathrm{A}}^{a} C_{\mathrm{I}}^{i} C_{\mathrm{X}}^{x}$

concentrations of a species formed during the process which inhibits the reaction. The slopes are given by eqns. (ii) to (iv).

$$
\mathrm{d} E^{\mathrm{p}} / \mathrm{d} \log v=[1 /(b+1)](\ln 10) R T / n F
$$

$\mathrm{d} E^{\mathfrak{p}} / \mathrm{d} \log C_{\mathrm{A}}=[(a+b+i-1) /(b+1)](\ln 10) R T / n F$

$\mathrm{d} E^{\mathrm{p}} / \mathrm{d} \log C_{\mathrm{X}}=[x /(b+1)](\ln 10) R T / n F$

The recognition that the origin of the responses lies in the reaction orders allows for the treatment of complex mechanisms for which numerical methods are not feasible as well as for competing mechanisms.

It has previously been shown that the linear sweep voltammetry (LSV) response; $\mathrm{d} E^{\mathrm{p}} / \mathrm{d} \log v$, $\mathrm{d} E^{\mathrm{p}} / \mathrm{d} \log C_{\mathrm{A}}, \mathrm{d} E^{\mathrm{p}} / \mathrm{d} \log C_{\mathrm{X}}$, can be predicted from generalized rules based on theoretical calculations. ${ }^{1}$ The voltage sweep rate $(v)$ dependence was predicted on the basis of the number of electrons transferred before or during the rate determining step of the process while the substrate concentration $\left(C_{\mathrm{A}}\right)$ dependence could be obtained from the rate equa- tion. The dependence of the peak potential on the concentration of an additional reactant $\left(C_{\mathrm{x}}\right)$ was concluded to be equal to $\mathrm{d} E^{\mathrm{p}} / \mathrm{d} \log v$ or 0 depending upon whether $X$ reacts before or after the rate determining step.

It has recently been pointed out that theoretical calculations of electrode mechanisms can only be carried out on relatively simple mechanisms without making severe approximations regarding to relative importance of some of the steps. ${ }^{2}$ The disproportionation mechanism (eqns. 1-3) illustrates this point. The rate law (4) must be treated as two $\mathrm{A} \pm \mathrm{e}^{-} \rightleftarrows \mathrm{B}$

$2 \mathrm{~B} \underset{k_{-2}}{\stackrel{k_{2}}{\rightleftarrows}} \mathrm{C}+\mathrm{A}$

$\mathrm{C}+\mathrm{X} \stackrel{k_{3}}{\rightarrow}$ product

limiting cases (5) and (6) depending upon the relative

Rate $=k_{3} K_{2} C_{\mathrm{B}}^{2} C_{\mathrm{x}} /\left(\left(k_{3} / k_{-2}\right) C_{\mathrm{X}}+C_{\mathrm{A}}\right)$

Rate $=k_{3} K_{2} C_{\mathrm{B}}^{2} C_{\mathrm{X}} / C_{\mathrm{A}}$

Rate $=k_{2} C_{\mathrm{B}}^{2}$

importance of the terms in the denominator of (4). The LSV slopes which have been reported for this mechanism ${ }^{3}$ have employed the above approximations and refer to the limiting cases.

A more general approach which would allow one to use LSV data for complex mechanisms for which satisfactory numerical solutions are not feasible is 
definitely needed. This is brought out clearly by the recent ${ }^{2,4}$ discussions which emphasize that electrode mechanisms are seldom simple. In this paper, a general approach which does not involve any calculations is suggested. The method follows from the fact that the response to a particular mechanism is a consequence of the reaction orders of the species involved.

\section{RESULTS AND DISCUSSION}

For the Nernstian electrode reaction (1) followed by homogeneous chemical reactions of intermediate $\mathrm{B}$, the shape and the position along the voltage axis of the current - potential curve is dependent upon the flux of A and B at the electrode surface. The fluxes in turn are dependent upon the kinetic behaviour of $A$ and $B$ which is dictated by the electrode mechanism. Since kinetic behaviour is best described in terms of a rate law, it follows that the fluxes should be relatable to some features of the rate law. The obvious features are the exponents of the concentration terms or the reaction orders. In the following discussion, each of the three LSV slopes is first related to the reaction orders and then the results are shown to be identical to those of numerical solutions of the kinetic-diffusion problem. In the discussion, the potential scale is taken to be the fundamental factor, $(\ln 10) R T / F$, which is equal to $59.2 \mathrm{mV}$ at $298 \mathrm{~K}$.

The electrode mechanism rate law. The obvious reaction orders of importance are those relating to $\mathrm{A}, \mathrm{B}$ and $\mathrm{X}$. In addition, a species may be formed during the reaction which affects the rate in the same way that regeneration of A does. Since the effect is usually an inhibiting one, these species are given the symbol $I$. Thus, for the case where rate constants are separable from the concentration terms, the rate law is given by (7), where the superscripts are the appropriate reaction orders.

Rate $=k_{\mathrm{obs}} C_{\mathrm{B}}^{b} C_{\mathrm{A}}^{a} C_{\mathrm{I}}^{i} C_{\mathrm{X}}^{x}$

Rate law (7) is for the case where the rate constants and concentration terms are separable. Numerical solutions are only available for mechanisms for which the latter is the case. More complex rate laws will be discussed later.

The voltage sweep rate and additional reactant dependence. The voltage sweep rate dependence is directly relatable to the reaction order in B $(b)$ by eqn. (8). Since the concentration of reactant $X$ can normally be controlled and kept in large excess, the dependence on this factor is simply the reaction order $(x)$ multiplied by the $v$ dependence as in (9).

$$
\mathrm{d} E^{\mathfrak{P}} / \mathrm{d} \log v=[(1 /(1+b)](\ln 10) R T / n F
$$

$$
\mathrm{d} E^{\mathrm{p}} / \mathrm{d} \log C_{\mathrm{X}}=[(\mathrm{x} /(1+b)](\ln 10) R T / n F
$$

The substrate concentration dependence. This factor is the most complicated of the LSV slopes. It depends upon reaction orders $b, a$ and $i$. The reason for the dependence on $i$ is that the concentration of species I will be directly dependent upon the total amount of reaction taking place which is governed by the substrate concentration, $C_{\mathrm{A}}$. The dependence of the peak potential on $C_{\mathrm{A}}$ is given by (10). Species $\mathrm{I}$ is frequently $\mathrm{H}^{+}$or $\mathrm{OH}^{-}$

Table 1. Electrode mechanisms and theoretical

\begin{tabular}{|c|c|c|c|c|}
\hline No. & Mechanism & $S_{1}^{b}$ & $S_{2}^{b}$ & $S_{3}^{b}$ \\
\hline 1 & $\mathrm{~B}+\mathrm{X} \stackrel{k}{\rightarrow} \mathrm{C}$ & $1 / 2$ & 0 & $1 / 2$ \\
\hline 2 & $\begin{array}{l}\mathrm{B}+\mathrm{X} \stackrel{k}{\rightarrow} \mathrm{C} \\
\mathrm{C}+\mathrm{B} \rightleftarrows \mathrm{D}+\mathrm{A}\end{array}$ & $1 / 2$ & 0 & $1 / 2$ \\
\hline 3 & $\begin{array}{l}2 \mathrm{~B} \stackrel{k}{\rightarrow} \mathrm{C}+\mathrm{A} \\
\mathrm{C} \rightarrow \mathrm{D}\end{array}$ & $1 / 3$ & $-1 / 3$ & 0 \\
\hline 4 & $\begin{array}{l}2 \mathrm{~B} \stackrel{K}{\rightleftarrows} \mathrm{C}+\mathrm{A} \\
\mathrm{C}+\mathrm{X} \stackrel{k}{\rightarrow} \mathrm{D}\end{array}$ & $1 / 3$ & 0 & $1 / 3$ \\
\hline 5 & $2 \mathrm{~B} \stackrel{k}{\rightarrow} \mathrm{C}$ & $1 / 3$ & $-1 / 3$ & 0 \\
\hline 6 & $\mathrm{~B}+\mathrm{A} \stackrel{k}{\rightarrow} \mathrm{C}$ & $1 / 2$ & $-1 / 2$ & 0 \\
\hline 7 & 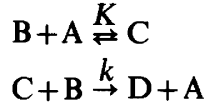 & $1 / 3$ & $-2 / 3$ & 0 \\
\hline 8 & $\begin{array}{l}\mathrm{B}+\mathrm{X} \stackrel{K}{\stackrel{K}{\rightleftarrows} \mathrm{C}+\mathrm{I}} \\
\mathrm{C}+\mathrm{B} \stackrel{k}{\rightarrow} \mathrm{D}\end{array}$ & $1 / 3$ & 0 & $1 / 3$ \\
\hline 9 & $\begin{array}{l}\mathrm{A} \pm 2 \mathrm{e}^{-} \rightleftarrows \mathrm{B}^{\prime} \\
\mathrm{B}^{\prime}+\mathrm{X} \stackrel{k}{\rightarrow} \mathrm{F}\end{array}$ & $1 / 4$ & 0 & $1 / 4$ \\
\hline 10 & $\mathrm{~B}^{\prime}+\mathrm{A} \stackrel{k}{\rightarrow} \mathrm{G}$ & $1 / 4$ & $-1 / 4$ & 0 \\
\hline 11 & $\begin{array}{l}\mathrm{B}^{\prime}+\mathrm{X} \stackrel{K}{\rightleftarrows} \mathrm{F}+\mathrm{I} \\
\mathrm{F} \stackrel{k}{\rightarrow} \mathrm{G}\end{array}$ & $1 / 4$ & $1 / 4$ & $1 / 4$ \\
\hline
\end{tabular}
LSV slopes. ${ }^{a}$

${ }^{a}$ Theoretical data from Refs. 3, 5-13. ${ }^{b} \mathrm{LSV}$ slopes in units of $(\ln 10) R T / n F$ as described in the text. 
Table 2. Linear sweep voltammetry slopes calculated from reaction orders. ${ }^{a}$

\begin{tabular}{lllllllll}
\hline Mechanism $^{b}$ & Rate & $b^{c}$ & $a^{c}$ & $i^{c}$ & $x^{c}$ & $S_{1}{ }^{d}$ & $S_{2}{ }^{d}$ & $S_{3}{ }^{d}$ \\
\hline 1 & $k C_{\mathrm{B}} C_{\mathbf{X}}$ & 1 & 0 & 0 & 1 & $1 / 2$ & 0 & $1 / 2$ \\
2 & $k C_{\mathrm{B}} C_{\mathbf{X}}$ & 1 & 0 & 0 & 1 & $1 / 2$ & 0 & $1 / 2$ \\
3 & $k C_{\mathrm{B}}^{2}$ & 2 & 0 & 0 & 0 & $1 / 3$ & $-1 / 3$ & 0 \\
4 & $k K C_{\mathrm{B}} C_{\mathrm{X}} / C_{\mathrm{A}}$ & 2 & -1 & 0 & 1 & $1 / 3$ & 0 & $1 / 3$ \\
5 & $k C_{\mathrm{B}}^{2}$ & 2 & 0 & 0 & 0 & $1 / 3$ & $-1 / 3$ & 0 \\
6 & $k C_{\mathrm{B}} C_{\mathrm{A}}$ & 1 & 1 & 0 & 0 & $1 / 2$ & $-1 / 2$ & 0 \\
7 & $k C_{\mathrm{B}}^{2} C_{\mathrm{A}}$ & 2 & 1 & 0 & 0 & $1 / 3$ & $-2 / 3$ & 0 \\
8 & $k K C_{\mathrm{B}} C_{\mathrm{X}} / C_{1}$ & 2 & 0 & -1 & 1 & $1 / 3$ & 0 & $1 / 3$ \\
9 & $k C_{\mathrm{B}}, C_{\mathrm{X}}$ & 1 & 0 & 0 & 1 & $1 / 4$ & 0 & $1 / 4$ \\
10 & $k C_{\mathrm{B}^{\prime}} C_{\mathrm{A}}$ & 1 & 1 & 0 & 0 & $1 / 4$ & $-1 / 4$ & 0 \\
11 & $k C_{\mathrm{B}^{\prime}} C_{\mathrm{X}} / C_{\mathbf{I}}$ & 1 & 0 & -1 & 0 & $1 / 4$ & $1 / 4$ & $1 / 4$ \\
\hline
\end{tabular}

${ }^{a}$ Calculated according to eqns. (8)-(10). ${ }^{b}$ As described in Table $1 .{ }^{c}$ Reaction orders as described in the text. ${ }^{d}$ LSV slopes in units of (ln 1) $R T / n F$ as described in the text.

$-\mathrm{d} E^{\mathrm{p}} / \mathrm{d} \log C_{\mathrm{A}}=$

$[(a+b+i-1) /(b+1)](\ln 10) R T / n F$

and in such cases $i$ can be eliminated from (10) by buffering the electrolyte solutions appropriately.

Applications to electrode mechanisms. The available data for LSV slopes calculated for various electrode mechanisms can be found in the classical paper by Nicholson and Shain, ${ }^{5}$ papers by Nicholson ${ }^{6,7}$ and the extensive work by Savéant and coworkers. ${ }^{8-13}$ In order to substantiate the validity of eqns. (8) $-(10)$, a number of mechanisms for which theoretical data is available are given in Table 1 along with the calculated LSV slopes indicated by $\mathrm{d} E^{\mathrm{p}} / \mathrm{d} \log v\left(S_{1}\right), \mathrm{d} E^{\mathrm{p}} / \mathrm{d} \log C_{\mathrm{A}}\left(S_{2}\right)$ and $\mathrm{d} E^{\mathrm{p}} / \mathrm{d} \log C_{\mathrm{X}}$ $\left(S_{3}\right)$. Applications of eqns. (8) $-(10)$ on the mechanisms, along with the corresponding rate equations, are given in Table 2 .

In all cases, eqns. (8) $-(10)$ correctly predict the values of $S_{1}, S_{2}$ and $S_{3}$. Although the list of mechanisms analyzed is not exhaustive, it encompasses all of the known types for which calculations have been carried out. Thus, it appears safe to conclude that eqns. (8) $-(10)$ give exact values of the slopes and that the relationship of the LSV response to reaction orders is valid.

Application to complex mechanisms. In more complex cases, all rate constants are not separable from all concentration terms as in (7). Thus, all reaction orders are not integers. Using the reaction order approach, it is possible to analyze complicated mechanisms and to make detailed mechanism assignments. To illustrate this, we can examine the disproportionation mechanism (eqns. 2 and 3) without making the limiting case approximations necessary in the numerical calculations of the response. From rate law (4) we can directly predict $\mathrm{d} E^{\mathrm{p}} / \mathrm{d} \log v$ and $\mathrm{d} E^{\mathrm{p}} / \mathrm{d} \log C_{\mathrm{x}}$ to be equal to $(1 / 3)$ (ln 10$) R T / F$ since the reaction orders $b$ and $x$ are equal to 2 and 1 , respectively, as in the limiting case (5). The complexity is manifested in the reaction order in substrate, $a$. The latter is dependent upon the relative magnitudes of the terms in the denominator of (4). From the observed values of $\mathrm{d} E^{\mathfrak{p}} / \mathrm{d} \log C_{\mathrm{A}}, a$ can be determined using the known values of $b$ and $x$ and eqn. (10). From the value of $a$ it would then be possible to estimate the rate constant ratio, $k_{3} / k_{-2}$. The observation of integral reaction orders in $B$ and $X$ and a non-integral value for $A$ should be sufficient to establish the form of the rate law.

In general, determination of $a, b, x$, and $i$ from experimental values of $S_{1}, S_{2}$ and $S_{3}$ should allow one to establish the form of the rate law for even very complex mechanisms.

Application to competing mechanisms. Another possible reason for the observation of non-integral reaction orders is that the process consists of competing reaction mechanisms. In this case the observed value of $S_{1}$ should be the most informative. If the reaction order in B differs for the two mechanisms, the value of $S_{1}$ will lie somewhere between the expected values for the two mechanisms. In this case, $b$ can be calculated directly from $S_{1}$ using eqn. (8). The relative importance of the two mechanisms is then obvious from the observed value of $b$.

Conclusions. The relationships between LSV slopes and reaction orders presented here allow the 
determination of the slopes directly from the rate equation for the electrode process. This not only eliminates the need to calculate the theoretical response for new mechanisms but also allows the LSV mechanism analysis to be extended to both complex mechanisms and to competing mechanisms. This represents an important advance from the viewpoint of the physical organic chemist interested in the study of reaction mechanisms of electrode generated intermediates. The recognition that the LSV slopes are direct consequences of the concept of reaction orders, familiar from homogeneous kinetics, removes some of the mystery associated with the technique caused by the practice of treating each new mechanism as a calculational objective.

\section{REFERENCES}

1. Parker, V. D. Acta Chem. Scand. B 34 (1980) 359.

2. Parker, V. D. Acta Chem. Scand. B 35 (1981) 233.

3. Mastragostino, M., Nadjo, L. and Savéant, J. M. Electrochim. Acta 13 (1968) 721.

4. Parker, V. D. Acta Chem. Scand. B 35 (1981) 123.

5. Nicholson, R. S. and Shain, I. Anal. Chem. 36 (1964) 706.

6. Nicholson, R. S. Anal. Chem. 37 (1965) 667.

7. Olmstead, M. L., Hamilton, R. G. and Nicholson, R. S. Anal. Chem. 41 (1969) 260.

8. Andrieux, C. P., Nadjo, L. and Savéant, J. M. J. Electroanal. Chem. 26 (1970) 147.

9. Andrieux, C. P., Nadjo, L. and Savéant, J. M. J. Electroanal. Chem. 42 (1973) 223.

10. Nadjo, L. and Savéant, J. M. J. Electroanal. Chem. 44 (1973) 327.

11. Nadjo, L. and Savéant, J. M. J. Electroanal. Chem. 48 (1973) 113.

12. Andrieux, C. P. and Savéant, J. M. J. Electroanal. Chem. 53 (1974) 165.

13. Ammar, F., Andrieux, C. P. and Savéant, J. M. J. Electroanal Chem. 53 (1974) 407.

Received January 8, 1981. 